\title{
First molecular detection and characterization of zoonotic Bartonella species in fleas infesting domestic animals in Tunisia
}

Saba Zouari, Fatma Khrouf, Youmna M'ghirbi and Ali Bouattour ${ }^{*}$

\begin{abstract}
Background: Bartonellosis is an emerging vector-borne disease caused by different intracellular bacteria of the genus Bartonella (Rhizobiales: Bartonellaceae) that is transmitted primarily by blood-sucking arthropods such as sandflies, ticks and fleas. In Tunisia, there are no data available identifying the vectors of Bartonella spp. In our research, we used molecular methods to detect and characterize Bartonella species circulating in fleas collected from domestic animals in several of the country's bioclimatic areas.

Results: A total of 2178 fleas were collected from 5 cats, 27 dogs, 34 sheep, and 41 goats at 22 sites located in Tunisia's five bioclimatic zones. The fleas were identified as: 1803 Ctenocephalides felis (83\%) (Siphonaptera: Pulicidae), 266 C. canis (12\%) and 109 Pulex irritans (5\%) (Siphonaptera: Pulicidae). Using conventional PCR, we screened the fleas for the presence of Bartonella spp., targeting the citrate synthase gene (g/tA). Bartonella DNA was detected in 14\% (121/866) of the tested flea pools [estimated infection rate (EIR) per 2 specimens: 0.072, 95\% confidence interval (Cl): 0.060-0.086]. The Bartonella infection rate per pool was broken down as follows: 55\% (65/118; EIR per 2 specimens: 0.329, 95\% Cl: 0. 262-0.402) in C. canis; 23.5\% (8/34; EIR per 2 specimens: 0.125 , 95\% Cl: 0.055-0.233) in P. irritans and 6.7\% (48/714; EIR per 2 specimens: $0.032,95 \%$ Cl: $0.025-0.045)$ in C. felis. Infection rates, which varied significantly by bioclimatic zone $(P<0.0001)$, were highest in the humid areas. By sequencing, targeting the gltA gene and the 16S-23S rRNA Intergenic Spacer Regions (ITS), we identified three Bartonella zoonotic species: B. elizabethae, B. henselae, B. clarridgeiae, as well as uncharacterized Bartonella genotypes.
\end{abstract}

Conclusions: To the best of our knowledge, this is the first time that fleas in Tunisia have been shown to carry zoonotic species of Bartonella. The dog flea, Ctenocephalides canis, should be considered the main potential vector of Bartonella. Our study not only provides new information about this vector, but also offers a public health update: medical practitioners and farmers in Tunisia should be apprised of the presence of Bartonella in fleas and implement preventive measures.

Keywords: Bartonella elizabethae, B. henselae, B. clarridgeiae, Ctenocephalides felis, C. canis, Pulex irritans, Bartonellosis, Zoonotic Bartonella species, Tunisia

\footnotetext{
*Correspondence: ali.bouattour@pasteur.rns.tn; bouattourali74@yahoo.com

Université de Tunis El Manar, Institut Pasteur de Tunis, Laboratoire

d'Epidémiologie et de Microbiologie Vétérinaire LR11IPT03, Service

d’Entomologie Médicale, 1002 Tunis-Belvédère, Tunisie
} 


\section{Background}

Global changes, including climate and land use, are among the most significant influences on the distribution and density of hematophagous arthropod species, which may affect zoonotic diseases including bartonellosis, caused by bacteria of the genus Bartonella (fastidious hemotropic Gram-negative, facultative intracellular organisms) belonging to the $\alpha 2$-subgroup of the proteobacteria. These organisms can infect animal and human erythrocytes and endothelial cells and cause polymorphic clinical diseases [1]. A host can be infected by Bartonella by transfusions/organ transplants, arthropod saliva (fleas, sand flies, lice and ticks) or by flea feces in the case of CatScratch-Disease (CSD) [1, 2].

Several tools that are useful and more sensitive than culture and serological techniques, including conventional PCR, RFLP, MST, MLVA and also the preenrichment culture using Bartonella-Alpha-Proteobacteria-Growth Medium combined with PCR and sequencing (ePCR) [3], have been developed to simultaneously detect and characterize Bartonella species and subspecies in hosts and vectors [4, 5]. More than 35 species and three subspecies have been identified to date, of which 17 species are involved or associated with an expanding spectrum of animal and human diseases. These include Carrion's disease, trench fever, bacillary angiomatosis, endocarditis and CSD $[6,7]$. CSD the most common zoonotic infection caused by Bartonella henselae, which is transmitted by cat fleas, Ctenocephalides felis. Bartonella henselae, which is frequently transmitted to humans by a cutaneous trauma (scratch and/ or bite) from an infected cat [8], was recently implicated in an intraocular inflammation caused by CSD in Tunisia [9]. Several cases of B. quintana endocarditis were also reported $[10,11]$.

Serological studies conducted in Tunisia showed canine seropositivity for $B$. vinsonii, $B$. henselae, B. clarridgeiae and $B$. bovis. This correlated well with tick and/or flea infestations of the dogs that were tested [12]. Moreover, a recent molecular study detected "Candidatus B. merieuxii” DNA in dogs [13]. While several clinical, molecular and serological studies have demonstrated that Bartonella species circulate among patients and dogs in Tunisia [8-10, 12], no data about the role of fleas as potential vectors are available.

This research used molecular methods to detect and characterize Bartonella species circulating in fleas collected from domestic animals and to compare its infection rates in Tunisia's various bioclimatic areas.

\section{Methods}

Flea collection and identification

An entomological investigation was carried out to collect fleas infesting domestic animals (dogs, cats, goats and sheep) in 22 sites located in Tunisia's five bioclimatic zones (humid, sub-humid, semi-arid, arid and Saharan, Fig. 1). Individual fleas were placed in labeled flasks and stored in $70 \%$ ethanol. All specimens were identified to species level using the standard taxonomic keys [14]. A distribution map of collected fleas, according to the bioclimatic areas, was made using ArcGIS 9 software (version 9.3.1).

\section{DNA extraction from fleas}

For DNA extraction, fleas from the same species, of the same sex, from the same host in the same site were pooled ( 2 fleas per pool, to reduce testing costs). The identified fleas were removed from the $70 \%$ ethanol flasks, washed, dried, and minced with disposable scalpels, and placed in $200 \mu \mathrm{l}$ ATL buffer. We followed the manufacturer's instructions for the QIAamp ${ }^{\oplus}$ DNA tissue extraction kit (Qiagen, Hilden, Germany). The final elution step was done using $90 \mu \mathrm{l}$ of $\mathrm{AE}$ buffer and then stored at $-20{ }^{\circ} \mathrm{C}$ for further use. To monitor for contamination during the processes, an extraction control (distilled water) for every 10 flea pools was added in each extraction experiment. The DNA concentration was determined with a NanoDrop spectrophotometer (NanoDrop ND-1000 Technologies, Wilmington, DE), and ranged between 50 and $400 \mathrm{ng} / \mu \mathrm{l}$. For the PCR reaction, the extracted DNA was diluted to reach a suitable concentration $(50 \mathrm{ng} / \mu \mathrm{l})$.

Detection of Bartonella spp. by PCR, DNA sequencing and phylogenetic relationships

Fleas were screened for the presence of Bartonella spp. using the citrate synthase gene ( $g l t A)$. The amplification of Bartonella DNA was carried out as described by Norman et al. [15] using the forward primer BhCS.7p (5'-GGG GAC CAG CTC ATG GTG G-3') and the reverse primer BhCS.1137n (5'-AAT GCA AAA AGA ACA GTA AAC A-3'), yielding a fragment of approximately 379 bp.

PCR amplifications were performed in a final volume of $25 \mu \mathrm{l}$ containing $2.5 \mu \mathrm{l}$ of $10 \times$ Taq buffer (Invitrogen, CA, USA), $1,5 \mu \mathrm{l}$ of $\mathrm{MgCl}_{2}$ (1.5 mM), $2.5 \mu \mathrm{l}$ of deoxyribonucleotide triphosphate $(200 \mu \mathrm{M}), 0.5 \mu \mathrm{l}$ of each primer (200 nM) and $0.75 \mathrm{U}$ of Taq Platinum polymerase (Invitrogen, CA, USA), followed by the addition of $3 \mu \mathrm{l}$ of flea DNA extracts. The amplification reaction was carried out in a thermocycler (Perkin Elmer 2400). PCR cycling conditions were $10 \mathrm{~min}$ at $94{ }^{\circ} \mathrm{C}$ for an initial denaturation, followed by 35 cycles of $30 \mathrm{~s}$ at $94{ }^{\circ} \mathrm{C}, 45 \mathrm{~s}$ at $51{ }^{\circ} \mathrm{C}$, and $30 \mathrm{~s}$ at $72{ }^{\circ} \mathrm{C}$, with a final extension of $7 \mathrm{~min}$ at $72{ }^{\circ} \mathrm{C}$. Positive and negative controls were included in each series of experiments. To avoid false-positive results, negative controls included sterile water and Bartonella-negative fleas DNA were added as the templates in each run. DNA 


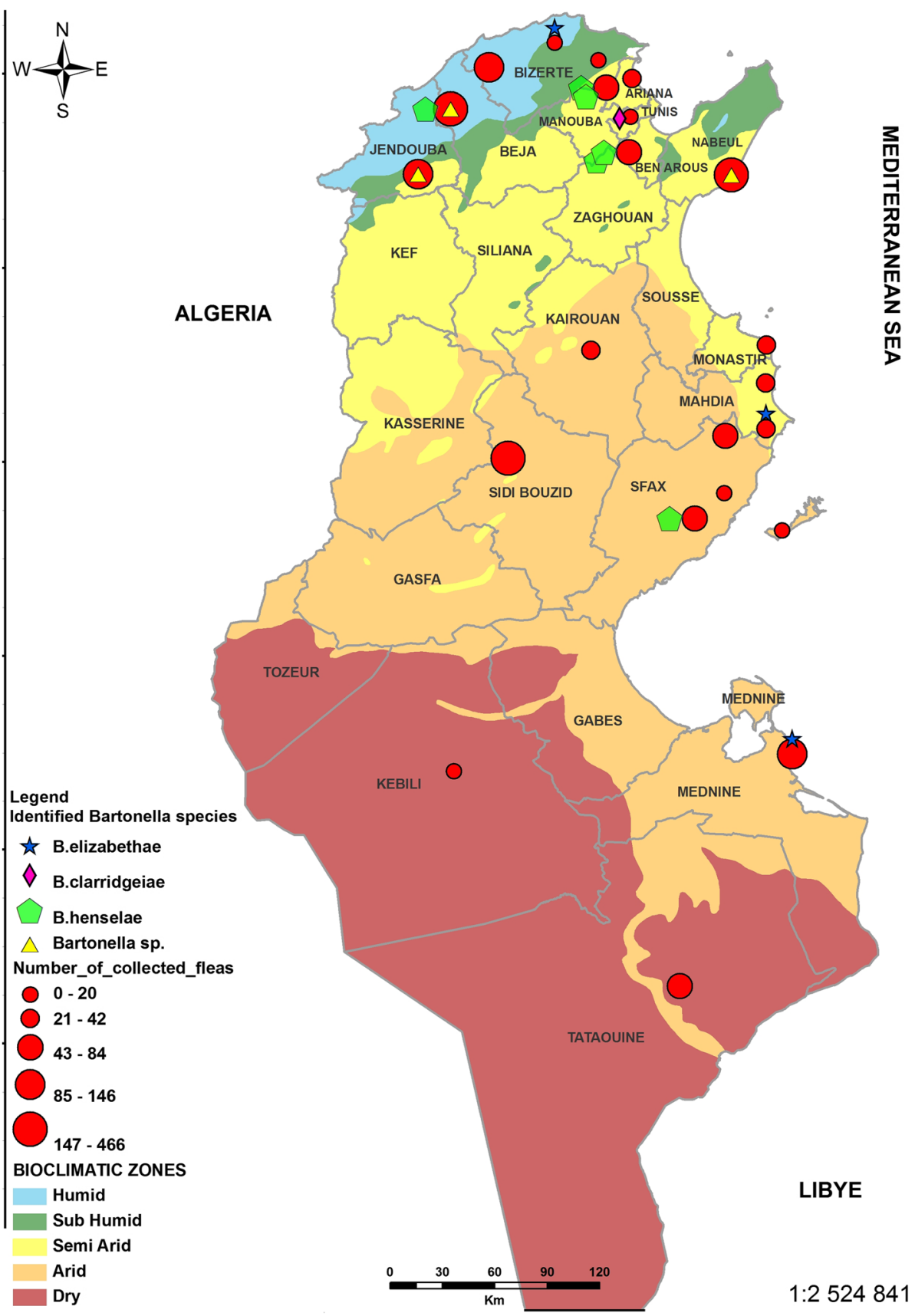

Fig. 1 Map of Tunisia showing the distribution of flea collection sites and repartition of Bartonella infected fleas according to bioclimatic areas

extracted from B. elizabethae culture (kindly provided by Pr. Zanzen, Hospital Hedi Chaker Sfax, Tunisia) was used as a positive control. To avoid cross-contamination, the extraction, amplification and gel electrophoresis were done in separate rooms. PCR products were visualized by ethidium bromide staining after electrophoresis in a 1.5\% agarose gel (Gellyphor, EuroClone, Milan, Italy) and their size was estimated by comparing them with Gene RulerTM 100-bp DNA Ladder (MBI Fermentas, Vilnius, Lithuania) as a molecular marker. PCR of the 16S-23S
rRNA Intergenic Spacer Regions (ITS) used the primers ITS2F (5'-GGG GCC GTA GCT CAG CTG-3') and ITS2R (5'-TGA ATA TAT CTT CTC TTC ACA ATT TC-3') [16]. For the ITS region, the PCR cycling conditions were performed as follows: $15 \mathrm{~min}$ at $94{ }^{\circ} \mathrm{C}$ for an initial denaturation followed by 35 cycles of 1 min at $94^{\circ}$ $\mathrm{C}, 35 \mathrm{~s}$ at $60{ }^{\circ} \mathrm{C}$, and $30 \mathrm{~s}$ at $72{ }^{\circ} \mathrm{C}$, with a final extension of $7 \mathrm{~min}$ at $72{ }^{\circ} \mathrm{C}$. PCR products were visualized by ethidium bromide staining after electrophoresis in a 3\% agarose gel (Gellyphor, EuroClone, Milan, Italy) and their size 
was estimated by comparing them with Gene RulerTM 50-bp DNA Ladder (Bio Basic Canada Inc., Markham, Canada). Gels were photographed using Gel Doc 2000 (Bio-Rad, Hercules, CA, USA).

All flea pools that had tested positive for the gltA Bartonella gene were confirmed by sequencing, targeting the two loci ( $g l t A$, ITS). Some PCR products were sequenced using the same oligonucleotides as for PCR to confirm the PCR results and to characterize the Bartonella species: PCR products were purified using the ExoSAP cleanup procedure (Amersham Biosciences, Piscataway, USA). All nucleotide sequences were obtained using the Big Dye Terminator v.3.1 Cycle Sequencing Kit (Applied Biosystems, Foster City, USA) and the 3130 automated sequencer (Applied Biosystems, Foster City, USA). Sequences were determined on both forward and reverse strands to maximize data accuracy for the two used loci. The glt $A$ and ITS sequences identified in this study were submitted to the GenBank database (see Availability of data and materials). Data sequences were compared and analyzed using the BLAST program (http:// www.ncbi.nlm.nih.gov/BLAST).

The CLUSTAL W algorithm was used for sequence alignments and MEGA 6.06 software for phylogenetic analyses. Sequences of the gltA gene were used to construct a phylogenetic tree using the neighbor-joining method.

\section{Statistical analysis}

Estimated infection rates (EIR) of all analyzed pools and of each flea species with corresponding 95\% confidence intervals $(\mathrm{CI})$ were calculated, based on equal pool sizes (2 fleas/tube), using the binGroup package [17] in the software R (R Core Team 2015).

The Chi-square test (EpiInfo 6.04) was used to compare the Bartonella infection rate of flea pools by species, animal host and bioclimatic area. The observed differences were considered significant when the resulting $P$-value was $<0.05$.

\section{Results}

\section{Collection and identification of fleas}

A total of 2178 fleas (67\% females, 33\% males) were collected from 5 cats $(n=51), 27$ dogs $(n=664)$, 34 sheep $(n=341)$ and 41 goats $(n=1122)$ from all sites in Tunisia where the investigation was carried out. The largest number of fleas was removed from goats. Ctenocephalides felis was found on all examined animals (cats, dogs, sheep and goats) and was the dominant species of those collected ( $83 \% ; n=1803)$, followed by Ctenocephalides canis $(12 \% ; n=266)$ and Pulex irritans (5\%; $n=109$ ) (Table 1).

A predominance of females was observed in the collected C. felis (1249 females, 554 males) and C. canis (158 females, 108 males), whereas for $P$. irritans males outnumbered females (60 males, 49 females).

Cats, sheep and goats were infested exclusively with $C$. felis, while dogs were infested with C. felis, C. canis and P. irritans (Table 1).

\section{PCR detection of Bartonella in collected fleas}

Among the 2178 collected fleas, 1732 specimens pooled in 866 tubes $(714 \mathrm{C}$. felis pools, $118 \mathrm{C}$. canis pools and $34 P$. irritans pools) were screened for the presence of Bartonella DNA, which was detected in 14\% (121/866) of the tested flea pools based on the gltA gene (EIR per 2 specimens: 0.072, 95\% CI: 0.060-0.086); details are summarized in Table 2. The overall Bartonella infection rates per pool of C. canis and P. irritans were 55\% (65/ 118 ) and $23.5 \%(8 / 34)$, respectively. For C. felis, only $6.7 \%(48 / 714)$ of the flea pool samples tested positive for Bartonella DNA (Table 2). The differences in the Bartonella infection rates per pool among the three flea species were statistically significant $\left(\chi^{2}=199.7 ; d f=2\right.$; $P<0.0001)$. The largest number of infected $C$. felis was removed from cats $(33.3 \% ; 8 / 24)$ and dogs $(16.6 \% ; 24 /$ 144). However, $5.8 \%(10 / 170)$ and $1.6 \%(6 / 376)$, of infected $C$. felis were collected from sheep and goats, respectively. The differences in numbers of infected $C$. felis observed by animal host were statistically significant $\left(\chi^{2}=65.7 ; d f=3 ; P<0.0001\right)$. The Bartonella infection rate per pool of all identified flea species was analyzed by the animal host: The rates in the flea pools collected from cats $(33.3 \%)$ and dogs $(32.7 \%)$ was higher than in those collected from sheep (5.8\%) and goats (1.6\%) (Table 1). These differences were statistically significant $\left(\chi^{2}=151.6 ; d f=3 ; P<0.0001\right)$. In this study we found

Table 1 The number of the identified flea species and Bartonella infection rate per flea pool by host species $(\mathrm{g} / \mathrm{t} A)$

\begin{tabular}{|c|c|c|c|c|c|}
\hline Flea species & Cat & Dog & Sheep & Goat & Total \\
\hline C. felis & 51 & 289 & 341 & 1122 & 1803 \\
\hline C. canis & 0 & 266 & 0 & 0 & 266 \\
\hline P. irritans & 0 & 109 & 0 & 0 & 109 \\
\hline Total & 51 & 664 & 341 & 1122 & 2178 \\
\hline $\begin{array}{l}\text { Bartonella infection rate per pool in } \\
\text { fleas } \%(X / Y)\end{array}$ & $\begin{array}{l}33.3 \\
(8 / 24)\end{array}$ & $\begin{array}{l}32.7 \\
(97 / 296)\end{array}$ & $\begin{array}{l}5.8 \\
(10 / 170)\end{array}$ & $\begin{array}{l}1.6 \\
(6 / 376)\end{array}$ & $\begin{array}{l}14 \\
(121 / 866)\end{array}$ \\
\hline
\end{tabular}

Abbreviations: $X$ number of pools positive for Bartonella DNA, $Y$ number of tested flea pools 
Table 2 The estimated infection rate of Bartonella over all analyzed pools and per each flea species with corresponding 95\% confidence intervals (Cl)

\begin{tabular}{lllllll}
\hline Flea species & Number of specimens & Pools tested & Positive pools & Estimated infection rate & $95 \% \mathrm{Cl}$ & Infection rate per pool (\%) \\
\hline C. felis & 1428 & 714 & 48 & 0.0342 & $0.0253-0.0450$ & 6.7 \\
C. canis & 236 & 118 & 65 & 0.3298 & $0.2628-0.4021$ & 55 \\
P. irritans & 68 & 34 & 8 & 0.1255 & $0.0552-0.2330$ & 23.5 \\
Total & 1732 & 866 & 121 & 0.0724 & $0.0604-0.0860$ & 14 \\
\hline
\end{tabular}

that fleas (C. felis, C. canis and P. irritans) infected with Bartonella were collected from 11 of the 22 sites in the different bioclimatic areas (Fig. 1), where the Bartonella infection rates of flea pools varied significantly. The highest infection rate was observed in the humid zone (28\%; 56/200), followed by the semi-arid (21.6\%; 54/ $249)$, sub-humid $(7 \% ; 4 / 57)$ and arid zones (2\%; 7/321). No Bartonella DNA was found in fleas collected in the Saharan areas $(0 \% ; 0 / 39)\left(\chi^{2}=67.13 ; d f=4 ; P<0.0001\right)$.

\section{Sequence analysis}

Only 81 out of 121 amplicons, obtained by targeting the gltA gene, were sequenced, while the remaining showed a weak positive signal on the electrophoresis gel. Sequencing made it possible to identify three zoonotic Bartonella species, B. henselae, B. elizabethae and $B$. clarridgeiae, as well as uncharacterized Bartonella genotypes. The number of sequencing samples and the distribution of Bartonella species detected in this study by bioclimatic zone, site, flea species, and animal host are summarized in Additional file 1: Table S1 and Table 3. Based on the gltA gene, a Bartonella sp. with an identity score between 98 and $100 \%$ with $B$. henselae isolated from human blood in France (GenBank: HG969191) was detected in 50 flea pool samples. A Bartonella sp. with $100 \%$ identity with $B$. elizabethae detected in dog ticks in Taiwan [18] (GenBank: GU056193), was detected in 8 flea pools. In addition, a Bartonella sp. with a 99\% identity score with $B$. clarridgeiae, described in Switzerland [19] (GenBank: FN645454), was detected in 4 flea pools. A Bartonella sp. with an identity score varying between 97 and 100\% with uncultured Bartonella sp. clone B224RnF isolated from rodents [20] (GenBank: KC763936), was detected in 8 flea pools. In addition, a Bartonella sp. with an identity score varying between 99 and $100 \%$ with Bartonella sp. BR10 detected in dog ticks in Taiwan [18] (GenBank: GU056200), was detected in 11 flea pools (Additional file 1: Table S1).
By sequencing the ITS region of 46 amplicons out of 81 , we were able to confirm the identification of the three zoonotic Bartonella species (B. henselae, B. elizabethae and $B$. clarridgeiae) detected by the gltA gene, with an identity score between 99 and $100 \%$ with the published sequences in GenBank (HG969191, L35103 and FN645454, respectively). In addition, a Bartonella sp. with an identity score of $100 \%$ with Bartonella sp. Lao/Nh2 isolated from rodents (GenBank: EU714977) [21], was detected in 8 flea pools that were identified as Uncultured Bartonella sp. B224RnF clones TUN by glt $A$ gene. Targeting the ITS region, we were unable to amplify and sequence the pools revealed positive for Uncultured Bartonella sp. BR 10 TUN clones by the gltA gene (Additional file 1: Table S1).

A gltA phylogenetic tree made it possible to visualize the arrangement of the Bartonella species identified in our study as compared to those identified in other studies. It showed that Tunisian sequences were clustered in 4 different groups (Fig. 2).

\section{Discussion}

Fleas are blood-sucking ectoparasites that are important vectors of pathogens including the bacteria of the Bartonella genus. In our study, 2178 fleas were removed from cats, dogs, sheep and goats from rural and urban sites in Tunisia's five bioclimatic zones. These animals spend most of their time outdoors exclusively. We observed a severe flea infestation in goats on several farms. Such highly infested animals can suffer from anemia and die as a result of untreated flea infestations, and create significant financial losses for their owners [22]. The collected fleas were identified as $C$. felis, $C$. canis and $P$. irritans. The cat flea, C. felis (83\% of all collected fleas), an ubiquist ectoparasite, was found on all investigated animal species in all regions. The same predominance has been observed in Germany, where Beck et al. [23] identified $81 \%$ of the fleas removed from dogs and cats as $C$. felis, followed by C. canis and P. irritans. A survey

Table 3 Number of Bartonella species, detected using the gltA gene, in the identified flea species collected in Tunisia

\begin{tabular}{lllll}
\hline Flea species & B. henselae & Uncharacterized Bartonella genotypes & B. elizabethae & B. clarridgeiae \\
\hline C. felis & 26 & 1 & 8 & 0 \\
C. canis & 24 & 10 & 0 & 0 \\
P. irritans & 0 & 8 & 0 & 0 \\
\hline
\end{tabular}




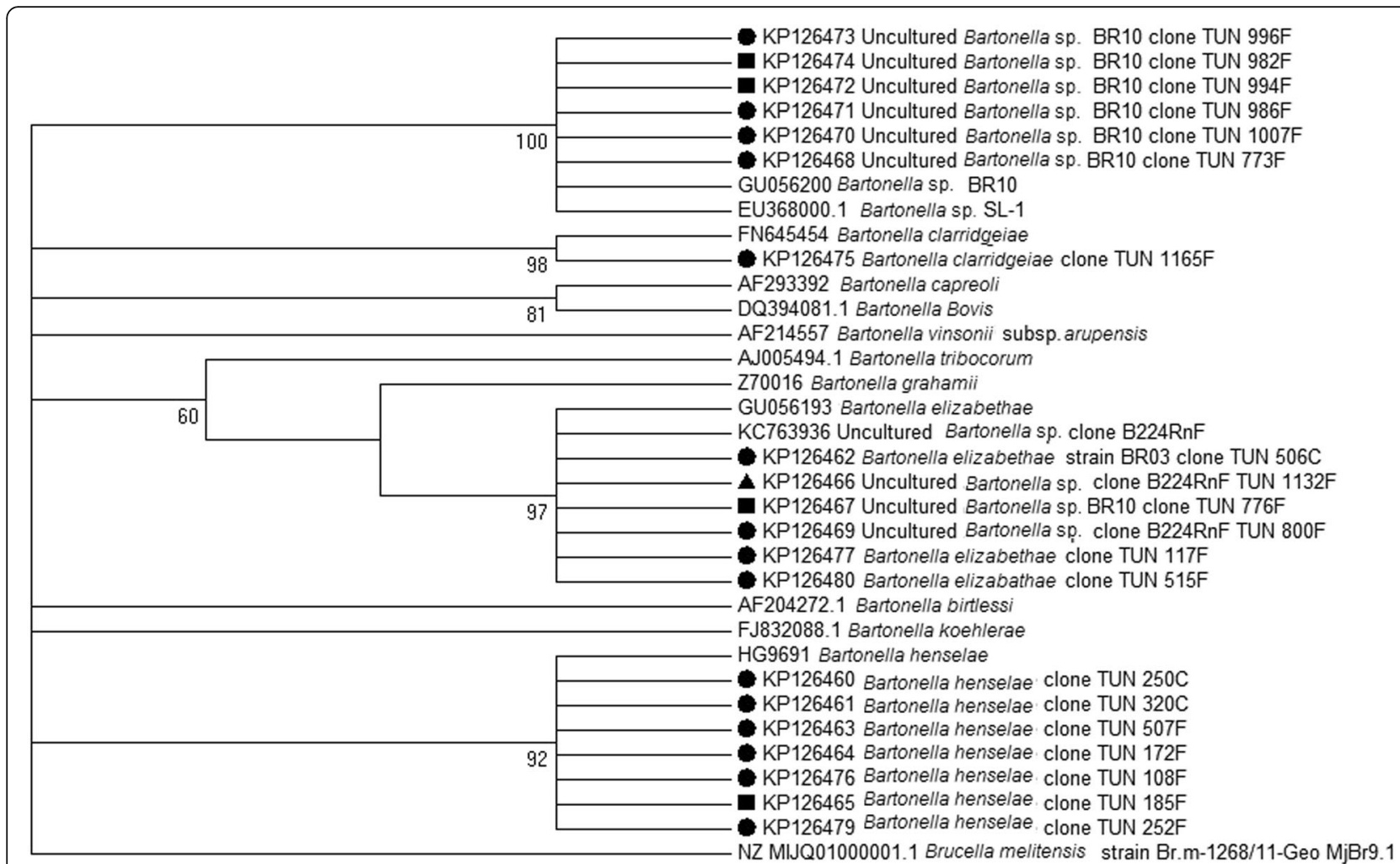

Fig. 2 Phylogenetic tree based on citrate synthase ( $g / t A)$ gene of Bartonella spp. using the MEGA 5.02 software. The tree was obtained using the neighbor-joining method. Numbers at the nodes are proportions of 1000 bootstrap resampling that support the topology. Brucella melitensis strain Br.m-1268/11-Geo MjBr9.1 was used as the outgroup to root the tree. TUN (Tunisia) sequences detected in this work have been deposited in GenBank under the accession numbers KP126460 - KP126480. • Ctenocephalides felis; $\mathbf{-}$ : Ctenocephalides canis; $\mathbf{\Delta}$ : Pulex irritans

conducted in Iran also showed $C$. felis to be the most frequent species infested dogs (42\%), followed by $P$. irritans (26.5\%) and C. canis (16.5\%) [24]. The same observations have been reported elsewhere in North Africa, in Egypt [25], Algeria [26], Libya [22] and Morocco [27]. Such data show the adaptation of cat fleas to other animal species. By contrast, $C$. canis collected from dogs was most prevalent in Ireland [28] and New Zealand [29].

Ctenocephalides canis and P. irritans were found only on dogs in northern Tunisia (Tunis, Béni Khiar, Jouza); $40.7 \%(11 / 27)$ had a mixed infestation of $C$. felis, C. canis and $P$. irritans (Table 1). Our finding is unsurprising, since $P$. irritans, which is normally associated with humans, has also been detected on dogs in Libya [22], Morocco [27], France [30] and Iran [24].

Our study showed that Bartonella DNA was detected in $14 \%$ of flea pools analyzed by the gltA gene. This rate is lower than those in studies from the UK (17\%; flea pools) [31] and France (26.2\%; single specimens) [32], but higher than those in studies from the USA (11.3\%; flea pools) [33], Israel (7.8\%; flea pools) [34], Hungary (4\%; flea pools) [35] and in countries neighboring Tunisia such as Morocco (4\%; single specimens) [27] and Algeria (9.33\%; single specimens). Our study showed variations in the Bartonella infection rates of flea pools in the different bioclimatic zones. The rates were highest in the humid area. No Bartonella infection was reported in the Saharan zone, confirming the results of a recent study undertaken in Tunisia showing that Bartonella seropositivity rates in dogs were highest in the humid zone [13].

The variation of Bartonella infection rates in countries, bioclimatic zones, and sites could be attributed to the detection methodologies used (culture or molecular approach), or to such bioclimatic parameters as temperature and humidity, the principal factors influencing flea survival, development, reproduction, and the abundance of Bartonella reservoirs. Fleas must be therefore carefully studied to better understand the relationships among climatic factors, Bartonella hosts and vectors $[24,36,37]$.

The Bartonella infection rates in the pools also vary with flea species: $55 \%$ for C. canis, $23.5 \%$ for P. irritans and $7 \%$ for C. felis. In Japan, all tested (single specimens) C. canis were infected with Bartonella compared to $33 \%$ in C. felis [38]. In Germany, the Bartonella infection rates of $C$. felis and C. canis were $2.4 \%$ and $0.4 \%$, respectively [32]. 
The positive PCR products were sequenced and analyzed in this study; thus, we were able to report for the first time in Tunisia that fleas harbor three known Bartonella species, B. elizabethae, B. henselae and B. clarridgeiae, as well as uncharacterized Bartonella genotypes. The first three species are zoonotic and were also reported in fleas in neighboring countries, where B. clarridgeiae and $B$. henselae were identified in $C$. felis collected from cats, goats and sheep in Morocco [27]. In Algeria, B. henselae, B. clarridgeiae and B. vinsonii berkhoffii were detected in $C$. felis and Xenopsylla cheopis collected from cats and dogs [26] whereas B. elizabethae, B.tribocorum and $B$. clarridgeiae were detected in fleas collected from hedgehogs, rats, and mice [39]. Based on the gltA gene and the ITS, we detected genotypes of uncultured Bartonella sp. (Bartonella sp. BR10 TUN, Uncultured Bartonella sp. clone B224RnF TUN and Bartonella sp. Lao/ Nh2 TUN). Bartonella sp. BR10 TUN was detected in $C$. felis and $C$. canis collected from dogs. This Bartonella species was also detected in fleas (Pulex simulans) and in brown dog ticks (Rhipicephalus sanguinus) collected from dogs in Taiwan and Costa Rica (Central America), respectively $[18,40]$. In our study, the DNA of uncultured Bartonella sp. clone B224RnF TUN (gltA) and uncultured Bartonella sp. Lao/Nh2 TUN (ITS) described in P. irritans collected from dogs were found in rodents in the USA and in Southeast Asia [20, 21].

The gltA phylogenetic tree shows that sequences of uncultured Bartonella sp. clone B224RnF TUN group in the same cluster with $B$. elizabethae TUN and very closely to B. tribocorum and B. grahamii, which are commonly detected in rodents and micromammals (Fig. 2) [20, 21]. This suggested that Bartonella sp. clone B224RnF TUN probably belongs to the rodent-borne pathogens. However, additional molecular markers are needed to identify the uncharacterized Bartonella genotypes. Bartonella species detected in fleas show that B. henselae (61.7\%) was most frequent followed by uncharacterized Bartonella genotypes (23.4\%), B. elizabethae $(9.8 \%)$ and B. clarridgeiae (4.9\%) (Additional file 1: Table S1, Table 3). We report that C. felis harbor different Bartonella species (B. henselae, B. elizabethae, B. clarridgeiae and uncharacterized Bartonella genotypes) while in C. canis we detected only B. henseale and uncharacterized Bartonella genotypes. Only uncharacterized Bartonella genotypes were detected in $P$. irritans (Table 3). The high frequency of $B$. henselae in our survey is unsurprising, given that it is considered to be the most common Bartonella species in infected fleas, mainly in C. felis [41]. In Morocco, B. henselae, the main agent of CSD, was also detected in $C$. felis collected from goats and sheep [27]. This species can be characterized by atypical symptoms and complications in 5-15\% of CSD patients who may have encephalitis, retinitis, osteitis, atypical pneumonitis, neurological syndromes, and prolonged fever [41]. Bartonella henselae can multiply in the digestive system of the cat flea and survive for several days in flea feces [8].

In Tunisia, Bartonella endocarditis seems to be common. Indeed, $9.8 \%$ of infective endocarditis was caused essentially by $B$. quintana, while $2.5 \%$ were caused by $B$. henselae $[9,10]$. The latest bacterium has also been involved in intraocular inflammation in Tunisian patients with CSD [9]. Humans are the principal, albeit not the sole, reservoir hosts for B. quintana, which was isolated from other hosts, including a cynomolgus monkey in Ireland [42] and dogs in New Zealand [43]. Endocarditis caused by $B$. quintana, which is a louse-borne pathogen, was associated with homelessness [44]. Recently, $B$. quintana DNA was detected in cat fleas, which means that this flea species may be an unrecognized vector for the transmission among cats of B. quintana [34]. It was also detected in the dental pulp of a cat in France [45] and was isolated in a cat and its owner in the USA [46].

Bartonella sp. was first reported in Tunisia in $49 \%$ of fat rats, Psammomys obesus, captured in the arid zone [46]. A molecular and serological study proved the presence of "Candidatus B. merieuxii", B. vinsonii, B. henselae, B. clarridgeiae and B. bovis, correlating with tick and/or flea infestations of examined dogs [12, 13]. Humans and dogs are accidental hosts of $B$. henselae, whereas cats are their natural reservoir [47]. Bartonella vinsonii berkhoffii, $B$. henselae and B. rochalimae are the main species known to infect canids [47]. Recently, many other Bartonella species, including B. elizabethae, have been detected in domestic dogs [48], confirming our finding insofar as fleas collected from dogs were positive for $B$. hensale and $B$. elizabethae. We also reported that small ruminants were infested with $C$. felis that were positive for $B$. elizabethae, a rodent-associated zoonotic Bartonella species. It is likely that dogs, sheep and goats were infected with $B$. elizabethae, or that the fleas had been exposed to infected rodents. B. elizabethae DNA was previously detected in dog blood in the USA [49] and in fleas collected from dogs in Israel [32]. To the best of our knowledge, this is the first report of $B$. elizabethae in fleas collected from sheep and goats. Infections caused by $B$. elizabethae in humans have been associated with endocarditis and neuroretinitis [50, 51].

Our results revealed that fleas infesting domestic cats were positive for $B$. clarridgeiae DNA. This species was first isolated in the USA, where a patient displayed worrying clinical signs six months after adopted an infested kitten [52]. Several studies report that C. felis could be a potential vector for $B$. clarridgeiae $[53,16]$, which was isolated from cats and dogs. Other surveys report that dogs living in rural areas and exposed to ticks and fleas are at greater risk of being infected with Bartonella spp.[54, 55]. 


\section{Conclusions}

Our study shows the presence of three flea species infesting domestic animals in Tunisia; Ctenocephalides felis was predominant in comparison to $C$. canis and $P$. irritans fleas. To our knowledge, this study has shown for the first time in Tunisia, that fleas carry three zoonotic Bartonella species, B. elizabethae, B. henselae and $B$. clarridgeiae, as well as uncharacterized Bartonella genotypes. Our results indicate that the dog flea, C. canis, should be considered to be the principal potential vector of Bartonella. The highest Bartonella infection rate of flea pools was recorded in the humid area of northern Tunisia. These data may open up prospects for further studies of the effect of bioclimatic factors on the biology of fleas and their infection by Bartonella. Extensive molecular studies are needed to identify the uncharacterized Bartonella genotypes. Given the high rate of Bartonella infection in fleas in Tunisia, medical practitioners, pet owners and farmers should be informed about the vectorial role of fleas in transmitting Bartonella.

\section{Additional file}

Additional file 1: Table S1. Distribution of Bartonella species by bioclimatic zones, site, flea species and animal host and partial sequencing analysis of gltA gene and the ITS region. (PDF $23 \mathrm{~kb}$ )

\section{Abbreviations}

Cl: Confidence interval; CSD: Cat-scratch disease; EIR: Estimated infection rate; gltA: citrate synthase gene; ITS: 16S-23S rRNA intergenic spacer region; MLVA: Multiple locus variable number tandem repeats analysis; MST: Multilocus sequence typing; NCBI: National Center for Biotechnology Information; RFLP: Restriction fragment length polymorphism

\begin{abstract}
Acknowledgements
The authors would in particular thank Adel Rhim and all farmers for their collaboration in collection of fleas. We are particularly grateful to Deborah Glassman, PhD, DC Washington USA, for her constructive comments and English corrections on the early drafts of the manuscript. We also thank $\mathrm{Pr}$ Abir Zanzen for giving us the Bartonella DNA positive control.
\end{abstract}

\section{Funding}

This work was financed by the IEVP coopération transfrontalière Italie-Tunisie 2007-2013 PROJET 2 PS1.3.023-RESTUS and the Tunisian Ministry for Higher Education, Scientific Research, and Technology.

\section{Availability of data and materials}

The data supporting the conclusions of this article are included within the article and its Additional file 1. Sequences generated in this study were deposited in the GenBank database under the following accession numbers: KP126460-KP126477, KP126479, KP126480 (gltA); MF066225-MF066237 (ITS)

\section{Authors' contributions}

SZ contributed to the collection and identification of fleas, performed molecular and data analysis, and drafted the manuscript. FK contributed to the collection and identification of fleas, molecular extraction and made the flea distribution map. YM supervised the molecular work and corrected the paper. $A B$ contributed to the design of work, collection of fleas and correction of the manuscript. All authors read and approved the final manuscript.

\section{Ethics approval and consent to participate}

This study is a part of the "PROJET 2 PS1.3.023-RESTUS" and has been approved by the Commission on Ethics and Animal Welfare of the Institut
Pasteur de Tunis - Université de Tunis El Manar-TUNISIA, number 2014/03/I/ LR11IPT03/N1. All technical procedures were in accordance with the National and the European legislation regarding animal welfare and have met the International Guiding Principles for Biomedical Research Involving Animals by the Council for the International Organizations of Medical Sciences.

\section{Consent for publication}

Not applicable.

\section{Competing interests}

The authors declare that they have no competing interests.

\section{Publisher's Note}

Springer Nature remains neutral with regard to jurisdictional claims in published maps and institutional affiliations.

Received: 1 June 2017 Accepted: 8 September 2017

Published online: 19 September 2017

\section{References}

1. Chomel BB, Boulouis H-J, Breitschwerdt EB, Kasten RW, Vayssier-Taussat M, Birtles RJ, et al. Ecological fitness and strategies of adaptation of Bartonella species to their hosts and vectors. Vet Res. 2009:40:29.

2. Regier Y, O'Rourke F, Kempf VAJ. Bartonella spp. - a chance to establish one health concepts in veterinary and human medicine. Parasit Vectors. 2016;9:261.

3. Cherry NA, Maggi RG, Cannedy AL, Breitschwerdt EB. PCR detection of Bartonella bovis and Bartonella henselae in the blood of beef cattle. Vet Microbiol. 2009;135:308-12.

4. Boulouis PH-J, Marignac G, Haddad N, Maillard R, Chomel B. Animal reservoirs and primary hosts of Bartonella. Bull Acad Veterinaire Fr. 2008:161:211-20.

5. Monteil M, Durand B, Bouchouicha R, Petit E, Chomel B, Arvand M, et al. Development of discriminatory multiple-locus variable number tandem repeat analysis for Bartonella henselae. Microbiol Read Engl. 2007;153:1141-8.

6. Breitschwerdt EB. Bartonellosis: one health perspectives for an emerging infectious disease. ILAR J. 2014;55:46-58.

7. Jiyipong T, Jittapalapong S, Morand S, Rolain J-M. Bartonella species in small mammals and their potential vectors in Asia. Asian Pac J Trop Biomed. 2014:4:757-67.

8. Chomel BB, Kasten RW, Floyd-Hawkins K, Chi B, Yamamoto K, RobertsWilson J, et al. Experimental transmission of Bartonella henselae by the cat flea. J Clin Microbiol. 1996:34:1952-6.

9. Ihem M-B, Errais K, Chebil A, Ouertani A. Cause rare de neurorétinite bilatérale: la maladie des griffes de chat. Rev Neurol (Paris). 2012;168:544-7.

10. Hammami R, Abid D, Abid L, Znazen A, Hentati M, Hammam A, et al. L'endocardite à Bartonella en Tunisie: particularités lésionnelles et évolutives. Pan Afr Med J. 2013;16:24-9.

11. Znazen A, Rolain J-M, Hammami N, Kammoun S, Hammami A, Raoult D. High prevalence of Bartonella quintana endocarditis in Sfax, Tunisia. Am J Trop Med Hyg. 2005;72:503-7.

12. Makhlouf S. Contribution à l'étude des bartonelloses chez le chien en Tunisie [veterinary thesis]: Ecole National de Medicine Véterinaire de Sidi Thabet; 2008.

13. Belkhiria J, Chomel BB, Ben Hamida T, Kasten RW, Stuckey MJ, Fleischman DA, et al. Prevalence and potential risk factors for Bartonella infection in Tunisian stray dogs. Vector Borne Zoonotic Dis. 2017;17:388-97.

14. Ménier K, Beaucournu JC. Taxonomic study of the genus Ctenocephalides Stiles \& Collins, 1930 (Insecta: Siphonaptera: Pulicidae) by using aedeagus characters. J Med Entomol. 1998:35:883-90.

15. Norman AF, Regnery R, Jameson P, Greene C, Krause DC. Differentiation of Bartonella-like isolates at the species level by PCR-restriction fragment length polymorphism in the citrate synthase gene. J Clin Microbiol. 1995;33:1797-803.

16. Varagnol M, Parola P, Jouan R, Beaucournu J-C, Rolain J-M, Raoult D. First detection of Rickettsia felis and Bartonella clarridgeiae in fleas from Laos. Clin Microbiol Infect. 2009;15(Suppl 2):334-5

17. Zhang B, Bilder C, Biggerstaff B, Schaarschmidt F. binGroup: Evaluation and experimental design for binomial group testing. 2012. https://cran.r-project. org/web/packages/binGroup/index.html. Accessed 17 Aug 2017.

18. Tsai Y-L, Lin C-C, Chomel BB, Chuang S-T, Tsai K-H, Wu W-J, et al. Bartonella infection in shelter cats and dogs and their ectoparasites. Vector Borne Zoonotic Dis. 2011;11:1023-30. 
19. Engel P, Salzburger W, Liesch M, Chang C-C, Maruyama S, Lanz C, et al. Parallel evolution of a type IV secretion system in radiating lineages of the host-restricted bacterial pathogen Bartonella. PLoS Genet. 2011;7:e1001296.

20. Hayman DTS, McDonald KD, Kosoy MY. Evolutionary history of rat-borne Bartonella: the importance of commensal rats in the dissemination of bacterial infections globally. Ecol Evol. 2013;3:3195-203.

21. Angelakis E, Khamphoukeo K, Grice D, Newton PN, Roux V, Aplin K, et al. Molecular detection of Bartonella species in rodents from the Lao PDR. Clin Microbiol Infect. 2009;15(Suppl 2):95-7.

22. Kaal JF, Baker K, Torgerson PR. Epidemiology of flea infestation of ruminants in Libya. Vet Parasitol. 2006;141:313-8.

23. Beck W, Boch K, Mackensen H, Wiegand B, Pfister K. Qualitative and quantitative observations on the flea population dynamics of dogs and cats in several areas of Germany. Vet Parasitol. 2006;137:130-6.

24. Tavassoli M, Ahmadi A, Imani A, Ahmadiara E, Javadi S, Hadian M. Survey of flea infestation in dogs in different geographical regions of Iran. Korean J Parasitol. 2010;48:145-9.

25. Amin OM. The fleas (Siphonaptera) of Egypt: distribution and seasonal dynamics of fleas infesting dogs in the Nile valley and delta. J Med Entomol. 1966;3:293-8.

26. Bessas A, Leulmi H, Bitam I, Zaidi S, Ait-Oudhia K, Raoult D, et al. Molecular evidence of vector-borne pathogens in dogs and cats and their ectoparasites in Algiers, Algeria. Comp Immunol Microbiol Infect Dis. 2016;45:23-8

27. Boudebouch N, Sarih M, Beaucournu JC, Amarouch H, Hassar M, Raoult D, et al. Bartonella clarridgeiae, B. henselae and Rickettsia felis in fleas from Morocco. Ann Trop Med Parasitol. 2011;105:493-8.

28. Baker KP, Hatch C. The species of fleas found on Dublin dogs. Vet Rec. 1972;91:151-2.

29. Guzman RF. A survey of cats and dogs for fleas: with particular reference to their role as intermediate hosts of Dipylidium caninum. N Z Vet J. 1984;32:71-3.

30. Franc $M$, Choquart $P$, Cadiergues MC. Species of fleas found on dogs in France. Rev Med Vet (Toulouse). 1998;149:135-40.

31. Shaw SE, Kenny MJ, Tasker S, Birtles RJ. Pathogen carriage by the cat flea Ctenocephalides felis (Bouché) in the United Kingdom. Vet Microbiol. 2004;102:183-8.

32. Just FT, Gilles J, Pradel I, Pfalzer S, Lengauer H, Hellmann K, et al. Molecular evidence for Bartonella spp. in cat and dog fleas from Germany and France. Zoonoses Public Health. 2008;55:514-20.

33. Yore K, DiGangi B, Brewer M, Balakrishnan N, Breitschwerdt EB, Lappin M. Flea species infesting dogs in Florida and Bartonella spp. prevalence rates. Vet Parasitol. 2014;199:225-9.

34. Sofer S, Gutiérrez R, Morick D, Mumcuoglu KY, Harrus S. Molecular detection of zoonotic bartonellae (B. henselae, B. elizabethae and B. rochalimae) in fleas collected from dogs in Israel. Med Vet Entomol. 2015;29:344-8.

35. Sréter-Lancz Z, Tornyai K, Széll Z, Sréter T, Márialigeti K. Bartonella infections in fleas (Siphonaptera: Pulicidae) and lack of bartonellae in ticks (Acari: Ixodidae) from Hungary. Folia Parasitol. 2006;53:313-6.

36. Silverman J, Rust MK, Reierson DA. Influence of temperature and humidity on survival and development of the cat flea, Ctenocephalides felis (Siphonaptera: Pulicidae). J Med Entomol. 1981;18:78-83.

37. Chinga-Alayo E, Huarcaya E, Nasarre C, del Aguila R, Llanos-Cuentas A. The influence of climate on the epidemiology of bartonellosis in Ancash, Peru. Trans R Soc Trop Med Hyg. 2004;98:116-24.

38. Ishida C, Tsuneoka H, lino H, Murakami K, Inokuma H, Ohnishi T, et al. Bartonella henselae infection in domestic cat and dog fleas. Kansenshogaku Zasshi. 2001;75:133-6.

39. Bitam I, Rolain JM, Nicolas V, Tsai Y-L, Parola P, Gundi VAKB, et al. A multigene analysis of diversity of Bartonella detected in fleas from Algeria. Comp Immunol Microbiol Infect Dis. 2012;35:71-6.

40. Rojas N, Troyo A, Castillo D, Gutierrez R, Harrus S. Molecular detection of Bartonella species in fleas collected from dogs and cats from Costa Rica. Vector Borne Zoonotic Dis. 2015;15:630-2.

41. Chomel BB, Boulouis HJ, Breitschwerdt EB. Cat scratch disease and other zoonotic Bartonella infections. J Am Vet Med Assoc. 2004;224:1270-9.

42. O'Rourke LG, Pitulle C, Hegarty BC, Kraycirik S, Killary KA, Grosenstein P, et al. Bartonella quintana in cynomolgus monkey (Macaca fascicularis). Emerg Infect Dis. 2005;11:1931-4.

43. Kelly P, Rolain J-M, Maggi R, Sontakke S, Keene B, Hunter S, et al. Bartonella quintana endocarditis in dogs. Emerg Infect Dis. 2006;12:1869-72.
44. Fournier P-E, Ndihokubwayo J-B, Guidran J, Kelly PJ, Raoult D. Human pathogens in body and head lice. Emerg Infect Dis. 2002;8:1515-8.

45. La VD, Tran-Hung L, Aboudharam G, Raoult D, Drancourt M. Bartonella quintana in domestic cat. Emerg Infect Dis. 2005;11:1287-9.

46. Breitschwerdt EB, Maggi RG, Sigmon B, Nicholson WL. Isolation of Bartonella quintana from a woman and a cat following putative bite transmission. J Clin Microbiol. 2007;45:270-2.

47. Chomel BB, Ermel RW, Kasten RW, Henn JB, Fleischman DA, Chang C-C. Experimental infection of dogs with various Bartonella species or subspecies isolated from their natural reservoir. Vet Microbiol. 2014;168:169-76.

48. Bai Y, Kosoy MY, Boonmar S, Sawatwong P, Sangmaneedet S, Peruski LF. Enrichment culture and molecular identification of diverse Bartonella species in stray dogs. Vet Microbiol. 2010;146:314-9.

49. Mexas AM, Hancock SI, Breitschwerdt EB. Bartonella henselae and Bartonella elizabethae as potential canine pathogens. J Clin Microbiol. 2002;40:4670-4.

50. Daly JS, Worthington MG, Brenner DJ, Moss CW, Hollis DG, Weyant RS, et al. Rochalimaea elizabethae sp. nov. isolated from a patient with endocarditis. J Clin Microbiol. 1993;31:872-81.

51. O'Halloran HS, Draud K, Minix M, Rivard AK, Pearson PA. Leber's neuroretinitis in a patient with serologic evidence of Bartonella elizabethae. Retina. 1998;18:276-8.

52. Clarridge JE 3rd, Raich TJ, Pirwani D, Simon B, Tsai L, Rodriguez-Barradas MC, et al. Strategy to detect and identify Bartonella species in routine clinical laboratory yields Bartonella henselae from human immunodeficiency virus-positive patient and unique Bartonella strain from his cat. J Clin Microbiol. 1995;33:2107-13.

53. Lappin M, Griffin B, Brunt J, Riley A, Burney D, Hawley J, et al. Prevalence of Bartonella species, haemoplasma species, Ehrlichia species, Anaplasma phagocytophilum, and Neorickettsia risticii DNA in the blood of cats and their fleas in the United States. J Feline Med Surg. 2006;8:85-90

54. Pappalardo BL, Correa MT, York CC, Peat CY, Breitschwerdt EB. Epidemiologic evaluation of the risk factors associated with exposure and seroreactivity to Bartonella vinsonii in dogs. Am J Vet Res. 1997;58:467-71.

55. Chomel BB, Kasten RW, Henn JB, Molia S. Bartonella infection in domestic cats and wild felids. Ann NY Acad Sci. 2006;1078:410-5.

\section{Submit your next manuscript to BioMed Central and we will help you at every step:}

- We accept pre-submission inquiries

- Our selector tool helps you to find the most relevant journal

- We provide round the clock customer support

- Convenient online submission

- Thorough peer review

- Inclusion in PubMed and all major indexing services

- Maximum visibility for your research

Submit your manuscript at www.biomedcentral.com/submit
Biomed Central 